\title{
Design improvement of automatic transmission system for remote controlled car
}

\begin{abstract}
Remote controlled cars currently in the market only offer one transmission mode. They are less efficient in acceleration while the torque cannot be increase during the low speed. Although improvement has been made by upgrading to two speed transmissions, but the gearing system is still not sufficient to maximize the speed of nitro engine. With a third gear, the car has the potential to increase its velocity and fully utilize its engine power. This paper focus on the transmission system model for 1/10th scale nitro engine which can produce up to $30,000 \mathrm{rpm}$. The third gears has shown significant improvement of the car theoretical speed up to $210 \mathrm{~km} / \mathrm{h}$.
\end{abstract}

Keyword: Remote control vehicle; Automatic transmission system; Centrifugal clutch 